\title{
Value-at-Risk and Tsallis statistics: risk analysis of the aerospace sector
}

\author{
Adriana P. Mattedi ${ }^{1}$, Fernando M. Ramos ${ }^{1}$, Reinaldo R. Rosa ${ }^{1}$ \\ Rosario N. Mantegna ${ }^{2}$ \\ ${ }^{1}$ Núcleo para Simulação e Análise de Sistemas Complexos - Laboratório \\ Associado de Computação e Matemática Aplicada - Instituto Nacional de \\ Pesquisas Espaciais (INPE) - São José dos Campos, Brazil \\ ${ }^{2}$ Istituto Nazionale per la Fisica della Materia, Unità di Palermo and \\ Dipartimento di Fisica e Tecnologie Relative, Università di Palermo, Viale delle \\ Scienze, I-90128, Palermo, Italia
}

\begin{abstract}
In this study, we analyze the aerospace stocks prices in order to characterize the sector behavior. The data analyzed cover the period from January 1987 to April 1999. We present a new index for the aerospace sector and we investigate the statistical characteristics of this index. Our results show that this index is well described by Tsallis distribution. We explore this result and modify the standard Value-atRisk (VaR), financial risk assessment methodology in order to reflect an asset which obeys Tsallis non-extensive statistics.
\end{abstract}

Key words: econophysics, aerospace sector, Tsallis statistics, Value-at-Risk PACS: 89.90.+n, 05.40,

\section{Introduction}

Financial institutions are subject to many sources of risk. Financial risk can be broadly defined as the degree of uncertainty about future net returns. Accurate evaluation of risks in financial markets is of crucial importance for the proper assessment and efficient mitigation of risk and, consequently, for optimal capital allocation. The increased volatility of financial markets during the last decade has induced the development of sophisticated risk management tools. In this context, Value-at-Risk (VaR) has become the standard measure that financial analysts use to quantify market risk. VaR is defined as the maximum potential loss in a portfolio value due to adverse market movements, for 
a given probability ( $5 \%$ or $1 \%$, for example) and a fixed time horizon (typically, 1 day). The great popularity achieved by this instrument is essentially due to its conceptual simplicity: VaR reduces the market risk associated with any portfolio to just one number, the loss associated to a given probability, under normal market conditions.

Here, we analyze the financial risk of the aerospace sector. The aerospace complex "weights" several hundreds of billions of dollars a year, and creates millions of high-wage, high skill jobs worldwide. Moreover, technologicallyintensive aerospace production generates extremely important positive spill over effects for the rest of the economy. However, this very fact - the need of large investments in advanced technologies - makes this industry highly susceptible to political and economic risks. For example, if we consider the

volatility as a risk measure, we remark that, in the last decade, the stocks of the main aerospace players presented a volatility $20 \%$ higher than that displayed by major financial indexes such as the Dow-Jones Industrial Index and the Standard \& Poor's 500 Index (see below). Since until presently, there is no single market indicator specially designed for tracking the performance of the aerospace sector as a whole, we introduce a new index, called Comprehensive AeroSpace Index (CASI), based on the stocks negotiated on the New York Exchange (NYSE) and the Over the Counter (OTC) markets. We investigate the statistical characteristics of the CASI and show that this index is well described by Tsallis distribution. We explore this result and modify the standard Value-at-Risk (VaR) risk assessment methodology in order to reflect an asset whose underlying dynamics obeys the non-extensive statistics.

This paper is organized as follows. Following this Introduction, in Section 2 we describe the data set used in this work, how the index CASI is computed, and which are its main statistical characteristics. In Section 3, we briefly introduce the VaR methodology, then generalize it to non normal, Tsallis market conditions, and apply this new methodology to the CASI time-series. Finally, our concluding remarks are presented in Section 4.

\section{The Comprehensive Aerospace Index (CASI)}

The data set used in this paper consists of twelve years of daily prices sets, from January, 1987 to April, 1999, from a comprehensive sample of leading aerospace-related companies with stocks negotiated on the New York Exchange (NYSE) and on the Over-the-Counter (OTC) markets. The number of aerospace companies included in the data set varied from 62 in 1987, to 146 in 1999. The CASI was calculated using a base-weighted aggregate methodology $[1,2]$ in order to reflect the total market value of each company. Following this 

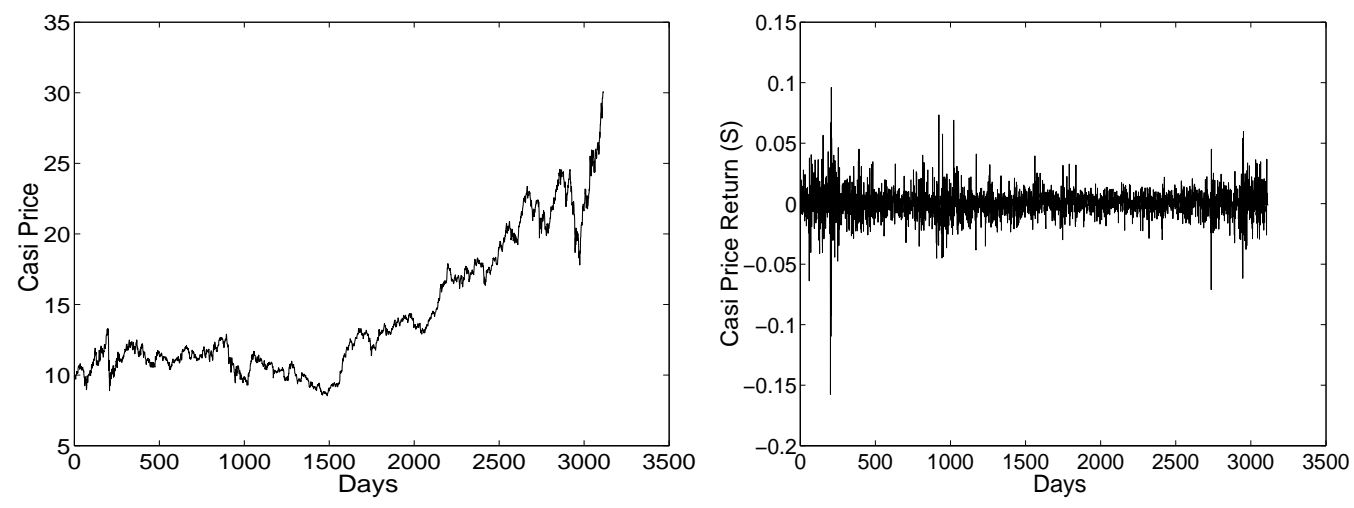

Fig. 1. The CASI index: (a) daily evolution; (b) daily returns.

approach, the CASI at a given time $t$ is given by

$$
Y(t)=\frac{1}{I D} \sum_{i}^{N}\left(\frac{w_{i}}{W}\right) X_{i}(t),
$$

where $w_{i}$ is the number of shares outstanding of the stock $i, W$ is the total number of companies shares that compose the index, $X_{i}$ is the price of the stock $i$, and $N$ is the number of stocks. ID is a normalizing factor called Index Divisor, which permits to set the initial CASI value to an arbitrary level (say, 100 or 10), allowing the index series to be comparable over time, and to adjust for variations in the weights $w_{i}$. These weights are up-dated every three months, or every time a difference of $5 \%$ or more in the number of shares outstanding of any stocks is detected or when a new stock is included in the index. In any case, the ID value is correspondingly adjusted to assure the continuity in the CASI index values.

As usual, we considered in our analysis the time evolution of CASI returns, defined as the logarithmic differences of CASI values over a period of time $\Delta t$, computed for a sequence of index values as $S_{\Delta t}(t)=\log (Y(t+\Delta t) / Y(t))$. CASI returns, from 1987 to 1999, are shown in Fig. 1, for a time delay of 1 day, together with the time evolution of the index itself during the same period. The spectral characteristics of both signals display some well-known features of financial time-series, with CASI returns behaving like a white-noise and the CASI index itself following a random-walk pattern. We present in Fig. 2, CASI return histograms (in semi-logarithmic plots), for three time intervals.

These results follow a familiar pattern already found in other financial signals $[3,4,5,6]$. The transition from an approximately Gaussian behavior, at long time intervals, to a leptokurtotic distribution form, as $\Delta t$ decreases, is quite evident. At $\Delta t=1$ day, the histogram has a kurtosis considerably higher than 3 , the value expected for a normal process. This feature, which corresponds to an excess of large fluctuations compared to a Gaussian distribution is a well 


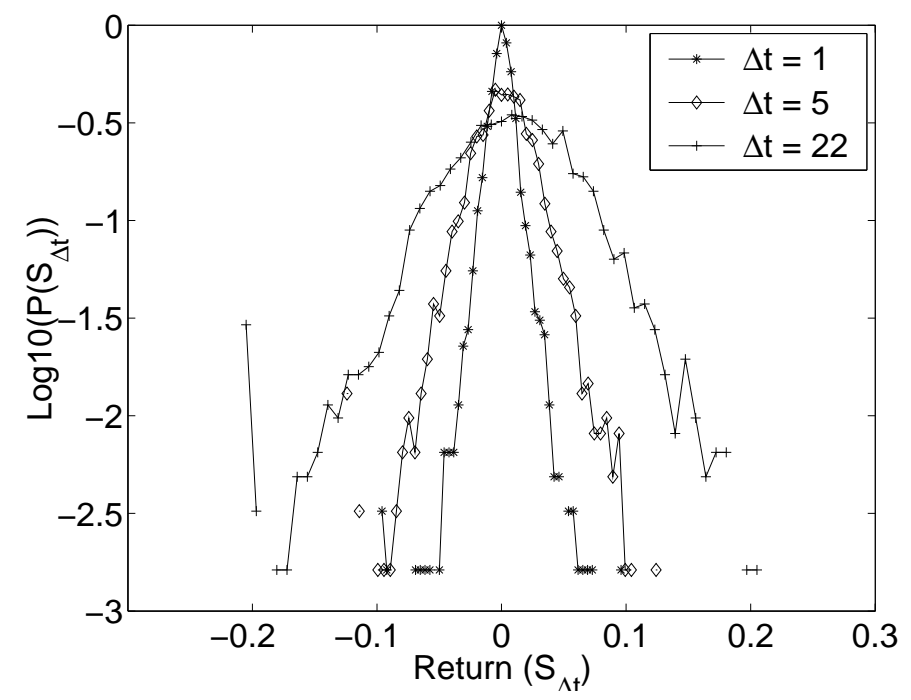

Fig. 2. CASI return histograms, for $\Delta t$ (1, 5, 22 days).

known signature of intermittence - bursts of intense market volatility.

Financial markets are extremely complex, with multiple hierarchical structures and a large number of heterogeneous agents interacting in an intricate way. When studying the statistical characteristics of a financial index, an usual approach is to investigate how the underlying distribution moments (or other related quantities) scale for different time intervals. In Fig. 3 we present, on a log-log plot, mean, variance and kurtosis as a function of $\Delta t$, for four financial indexes: CASI, Nasdaq, Dow-Jones Industrial (DJI) and Standard \& Poor's (S\&P500). We remark that all curves present a power-law behavior (the corresponding scaling exponents are displayed in Table 1), although the kurtosis results suffer from the finiteness of the sample size. For all indexes, the variance scales linearly with $\Delta t$, the expected result for a standard diffusive regime. Note that CASI and Nasdaq have kurtosis exponents quite different from those displayed by DJI and S\&P500. We may speculate that this result indicates that the dynamics of price formation in technology-intensive companies is peculiar, very different from more traditional industries. Surprisingly, we find that, although CASI and Nasdaq consistently display higher variances,

Table 1: Scaling coefficients

\begin{tabular}{ccccc}
\hline \multicolumn{5}{c}{ Indexes } \\
\hline & CASI & S\&P500 & DJI & Nasdaq \\
\hline Mean & 0.96 & 0.99 & 0.96 & 0.98 \\
\hline Variance & 1.00 & 0.93 & 0.96 & 1.09 \\
\hline Kurtosis & -0.38 & -0.58 & -0.61 & -0.32 \\
\hline
\end{tabular}


their kurtosis are smaller. In other words, CASI and Nasdaq are more volatile but less intermittent than DJI and S\&P500. This somewhat unexpected result suggests the presence of long memory effects in CASI and Nasdaq volatility patterns. These correlations tend to cluster periods of similar volatility, preventing the excessive alternating of price changes regimes.

\section{Risk Analysis}

In recent years, VaR has been adopted as the standard tool used by institutions to measure and manage financial market risk. Moreover, VaR is the basis for BIS (Bank for International Settlements) market risk-based capital requirements $[7,8]$. Although, its origins can be trace back as far as 1922, to capital requirements the NYSE (New York Stock Exchange) imposed to member firms, passing through the portfolio theory and a first crude VaR measure published in 1963 [9], VaR only became widely used with the J.P.Morgans RiskMetrics Technical Document in 1995 (freely available on the J.P.Morgan's website [10]). Great part of its popularity originates from the fact it aggregates several risk components into a single number. VaR measures the worst expected loss under normal market conditions over a specific time interval (which may vary from hours to years), at a given confidence level (for example, $99 \%$ or $95 \%$ ).

In general terms, VaR measures the quantile of the projected distribution of gains and losses over a given time horizon. The $q$-quantile (or fractile) of a random variable $X$ is any value $x$ such that $\operatorname{Pr}(X \leq x)=q$. If $c$ is the selected confidence level, VaR is the corresponding $1-c$ lower-tail quantile. Analytically, it can be formulated in terms of returns of the portfolio as follows:

$$
\operatorname{Pr}\left[S_{\Delta t}<\operatorname{VaR}_{S}(\Delta t)\right]=1-c,
$$

where $\operatorname{VaR}_{S}(\Delta t)$ is the VaR, in terms of returns, for a time horizon of $\Delta t$.

It is clear from Eq. (2), that the precise prediction of the probability of an extreme movement in the value of a portfolio is essential for risk management. By their very nature, extreme movements are related to the tails of the distribution of the underlying data generating process. Several studies, starting with the pioneering work by Mandelbrot [11], have shown that financial return distributions are leptokurtotic, that is they have heavier tails and a higher peak than a normal distribution. These features are well illustrated by CASI 1-day return distribution, shown in Fig. 2. In practical terms, the assumption of normally distributed returns may lead to serious errors in the estimation of risk, affecting the stability of markets and/or the profitability of financial institutions. 

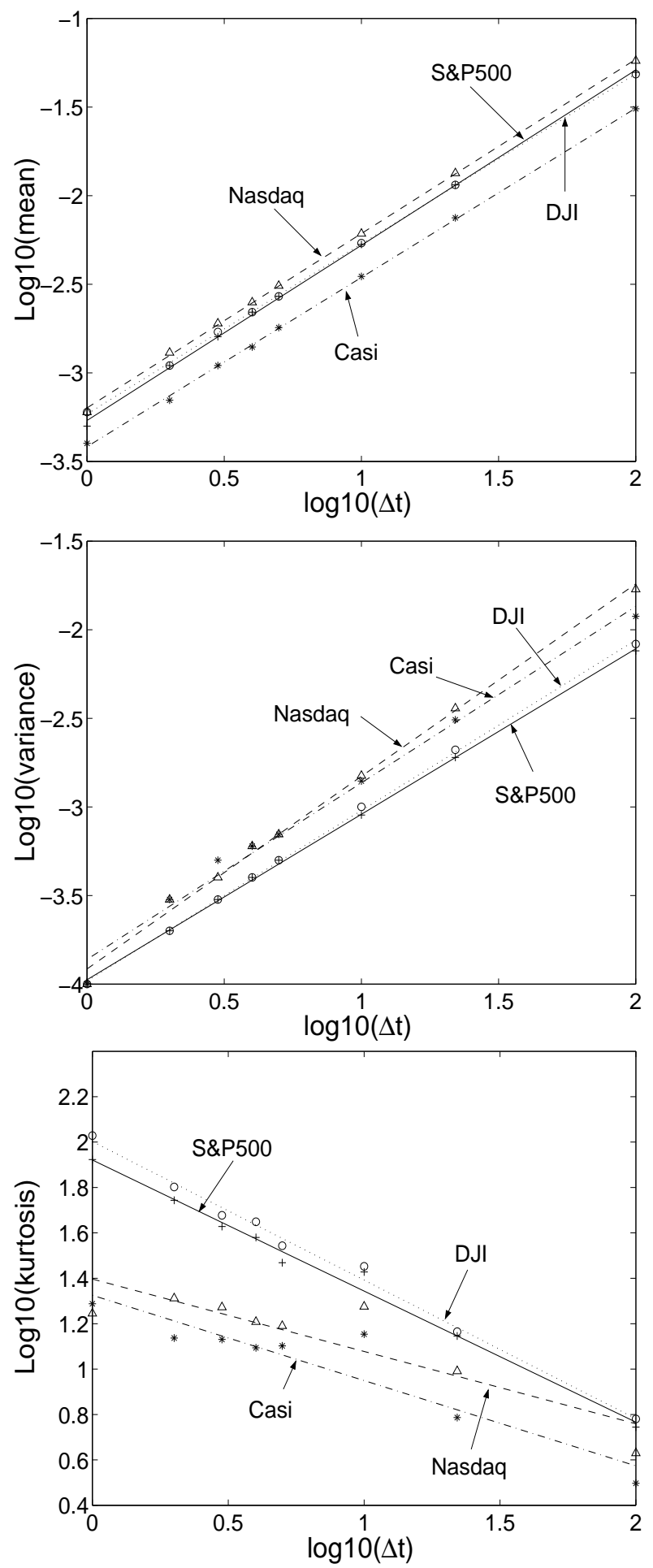

Fig. 3. Scaling with varying time intervals of CASI, Nasdaq, DJI and S\&P500: (a) mean; (b) variance; (c) kurtosis.

In order to model fat-tailed distributions, the log-normal distribution, generalized error distribution, truncated Levy distribution, and mixtures of normal distributions have been suggested $[12,13,14,15,16]$. From an econophysics 
point-of-view, several studies pointed out that the financial market dynamics may efficiently be understood within the statistical mechanical framework and its suitable generalizations $[14,15]$. In this context, Ramos and collaborators $[13,5]$ have recently shown that Tsallis canonical distribution [17], given by

$$
p_{q}(x) \sim\left(1-\beta(1-q) x^{2}\right)^{1 /(1-q)}
$$

with $q$ and $\beta$ as parameters, provides a simple and accurate model for financial histograms. Moreover, Tsallis entropic parameter $q$ was found to be an excellent measure of intermittency [18]. Note that, for $1<q<5 / 3$, Eq. (3) displays fat-tails but still has finite variance. For $q=1$, we recover the Gaussian distribution. Finally, for unit variance, we have $\beta=1 /(5-3 q)$.

Relaxing the assumption of normal returns intrinsic to the original VaR method, and assuming that market return distributions follow Eq. (3), $\operatorname{VaR}_{S}(\Delta t)$ can be computed from

$$
Z_{q}^{-1} \int_{-\infty}^{-V a R_{S}(\Delta t)}\left[1-\beta(1-q) S^{2}\right]^{1 /(1-q)} d S=1-c
$$

where $q$ is a function of $\Delta t$ and, for unit variance, $\beta=1 /(5-3 q)$. The normalizing factor is given by

$$
Z_{q}=\sqrt{\frac{\pi}{\beta(q-1)}} \frac{\Gamma((3-q) / 2(q-1))}{\Gamma(1 /(q-1))} .
$$

It is important to remark that, since the entropic parameter $q$ only depends on the kurtosis of the experimental histogram [18], its value can be easily estimated for longer time horizons. For this, it suffices to extrapolate the kurtosis value of the 1-day histogram, assuming some known scaling with $\Delta t$, as suggested by Fig. 3c.

Figure 4a compares two theoretical cumulative density functions (CDF), computed using Gaussian and Tsallis $(q=1.51)$ standardized return distributions, with CASI 1-day experimental histograms. We observe that Tsallis distribution provides better VaR estimates, for confidence levels within the range of 95 to $99.5 \%$. This comparison may be further enhanced plotting the difference between experimental and theoretical quantiles as a function of the confidence level (4b). Note that for high confidence levels (above 99\%), the Gaussian model grossly underestimates the risk, while for low confidence levels (below 97\%), it leads to sub-optimal capital allocation due to systematic risk overestimation. 

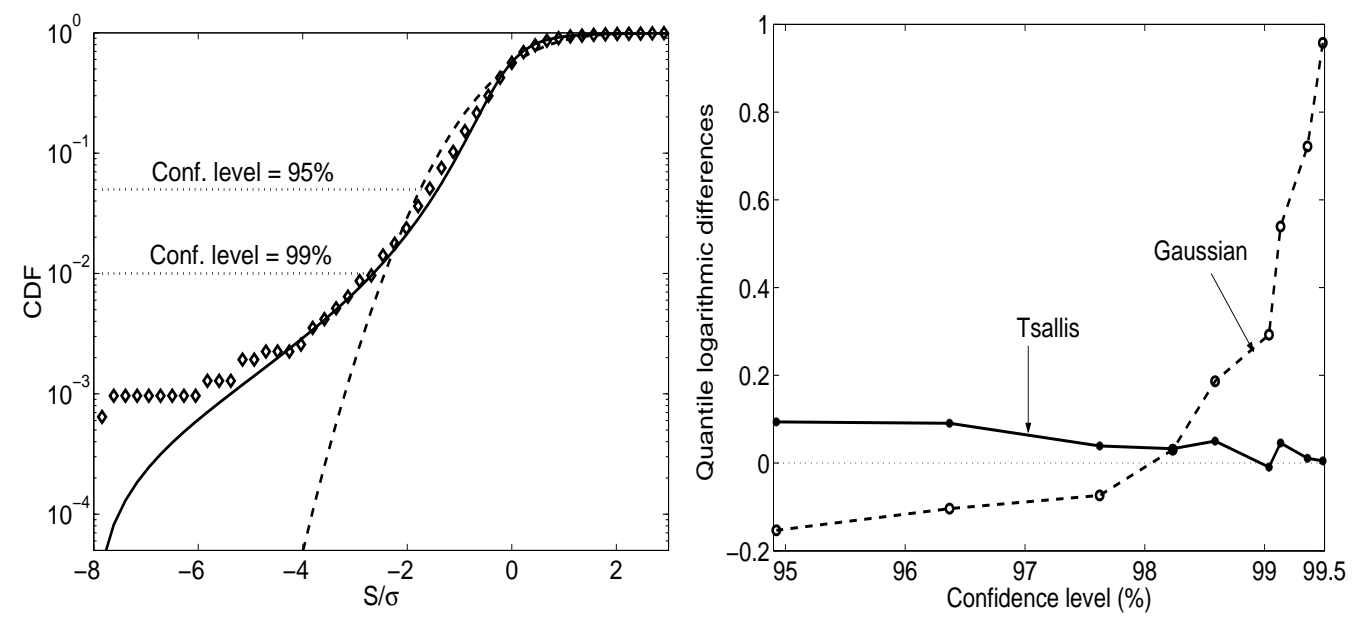

Fig. 4. (a) Experimental $(\diamond)$ and theoretical (Gaussian: dashed line; Tsallis: solid line, $q=1.51)$ cumulative density functions (CDF); (b) Experimental and theoretical quantile logarithmic differences as a function of the confidence level.

\section{Conclusion}

In this paper, we studied the financial risk of the aerospace sector. In spite of its undeniable economic, social and even strategic importance, until presently, there was no single market indicator specially designed for tracking the performance of the aerospace sector as a whole. This fact led us to develop a new index, called Comprehensive Aerospace Index (CASI), based on the stocks negotiated on the New York Exchange (NYSE) and the Over the Counter (OTC) markets. Our data set consisted of twelve years of daily prices sets, from January, 1987 to April, 1999, from a comprehensive sample of leading aerospace-related companies.

We investigated the statistical characteristics of the CASI and found that it is more volatile but less intermittent than other traditional market indicators, such as the DJI and S\&P500. This results suggests the existence of long memory correlations impacting the volatility clustering patterns of the CASI index. We also shown that CASI histograms, for different time intervals, are well described by Tsallis canonical distribution. We explored this result and modified the standard Value-at-Risk (VaR) risk assessment methodology in order to reflect an asset whose underlying dynamics obeys Tsallis non-extensive statistics. Our results have shown this approach provides better VaR estimates, for confidence levels within the range of 95 to $99.5 \%$.

Summarizing, we explored in this paper the idea that the comprehension of financial time-series statistical features may be the first step to grasp the behavior of a highly complex system such the financial market. Investment professionals have at their disposal a wide array of sophisticated tools to aid their analyzes, like the Value-at-Risk method. Many of these tools depend 
on the assumption that the asset returns are normally distributed. Today we know they are not. To emphasize the importance of this point, we showed in our results that the relaxation of normality premise can improve a risk measure, with positive impacts on the stability of markets and the profitability of financial institutions.

\section{Acknowledgments}

This work was supported by FAPESP, CAPES and CNPq, Brazil.

\section{References}

[1] New York Stock Exchange, Nyse Composite Index methodology guide. Jan. (2003).

[2] Standard \& Poor's., SESP analysts' handbook - 3o. chapter (1999).

[3] R.N. Mantegna and H.E. Stanley, Nature 376, 46-49 (1995).

[4] S. Ghashghaie, W. Breymann, J. Peinke, P. Talkner and Y. Dodge, Nature 381, 767-770 (1996).

[5] F.M. Ramos, R.R. Rosa, C. Rodrigues Neto, J.A. Bolzan, L.D.A. Sá, NonLinear Analysis 47,3521 (2001).

[6] A.P. Mattedi, F.M. Ramos, R.R. Rosa, M.M. De Marchi, L.S. Bins. Anais do XXXIV Simpósio Brasileiro de Pesquisa Operacional. ISSN 1518-1731 (2002).

[7] P. Jorion, Value at Risk: the new benchmark for controlling market risk. New York: McGraw-Hill Companies Inc.(1997).

[8] A. Saunders, Credit Risk Measurement: new approaches to Value at Risk and other paradigms. John Wiley \& Sons Inc., New York, (1999).

[9] G.A. Holton, Contigency Analysis, Working Papers http://econwpa.wustl.edu/eps/mhet/papers/0207/0207001.pdf (2002).

[10] J.P.Morgan. RiskMetrics: technical Document. Fourth Edition, New York, (1996).

[11] B. Mandelbrot, The Journal of Business 36, 394-419, (1963).

[12] R.N. Mantegna and H.E. Stanley, An Introduction to Econophysics: Correlations and Complexity in Finance,(Cambridge Univ. Press, Cambridge UK,2000). 
[13] F.M. Ramos, C. Rodrigues Neto, R.R. Rosa, Preprint cond-mat/9907348 (1999).

[14] F. Michael and M.D. Johnson, cond-mat/0108017 (2001).

[15] M. Ausloos and K. Ivanova. Phys. Rev. E 68, 46122 (2003).

[16] Y. Liu, P. Gopikrishnan, P. Cioeau, M. Meyer, C.-K.Peng, H.E. Stanley, Phys. Rev. E 60, 2, 1399 (1999).

[17] C. Tsallis, J.Stat.Phys. 52 (1988).

[18] J.A. Bolzan, F.M. Ramos, L.D.A. Sá, C. Rodrigues Neto, R.R. Rosh, J. Geoph. Res. 107, 8063, (2002). 